\title{
Desafios e inovações no uso de ferramentas tecnológicas para a vigilância epidemiológica em tempos de Covid-19
}

\author{
Challenges and innovations in the use of technological tools for epidemiological \\ surveillance in Covid-19 times \\ Retos e innovaciones en el uso de herramientas tecnológicas para la vigilancia \\ epidemiológica en tiempos de Covid-19
}

Pollyana Rocha de Araujo1*, Lígia Maria Cabedo Rodrigues ${ }^{1}$, Sandra Maria Gomes de Sousa1, Dânia Lima Cruz¹, Francisca Sandra Fortes Sampaio ${ }^{1}$, Silvio Cesar dos Santos ${ }^{1}$, Maria Teresa da Silva Ferreira ${ }^{1}$, Juliana de Menezes Dantas ${ }^{1}$, Larissa Santos da Silva ${ }^{1}$. Paula Roberta Silva Magalhães ${ }^{1}$.

\section{RESUMO}

Objetivo: Descrever a utilização de ferramentas tecnológicas por um Serviço de Vigilância Epidemiológica Hospitalar no contexto da Covid-19 em um Hospital Universitário do Nordeste do Brasil. Relato de experiência: O Hospital Universitário do Piauí tornou-se referência para Covid-19 desde abril de 2020. A vigilância epidemiológica utilizou a coleta de dados através do prontuário eletrônico e a comunicação com os pacientes e familiares por ligações telefônicas para a realização da notificação dos casos. Para o repasse das informações aos órgãos oficiais, fez-se uso de ferramentas tecnológicas como o E-SUS VE e o Sivep Gripe. Para fins de acesso a informações disponíveis para os profissionais da instituição, utilizou-se a intranet através do uso do Sisah, dispositivo através do qual foi possível disseminar as informações relativas a Notas Técnicas oficiais e atualizadas do Ministério da Saúde. Considerações Finais: Os registros eletrônicos e ferramentas tecnológicas utilizadas na saúde têm grande potencial para melhorar os cuidados, facilitando a transmissão rápida e precisa de dados do paciente, razão de extrema relevância para as ações de vigilância epidemiológica da Covid-19.

Palavras-chave: Covid-19, Notificação, Inovação, Vigilância epidemiológica.

\begin{abstract}
Objective: To describe the use of technological tools by a Hospital Epidemiological Surveillance Service in the context of Covid-19 in a University Hospital in Northeast Brazil. Experience report: The Hospital Universitário do Piauí has become a reference for Covid-19 since April 2020. An epidemiological surveillance uses data collection through electronic medical records and communication with patients and family members by telephone calls to perform the notification of the cases. For the transfer of information to official bodies, technological tools such as E-SUS VE and Sivep Gripe were used. For the purpose of accessing the information available to the institution's professionals, an intranet is used using Sisah, a device through which it was possible to disseminate information related to official and updated Technical Notes from the Ministry of Health. Final Considerations: The records electronics and technological tools used in healthcare have great potential to improve care, facilitating the fast and accurate transmission of patient data, an extremely important reason for covid-19's epidemiological surveillance actions.
\end{abstract}

Key words: Covid-19, Notification, Innovation, Epidemiological monitoring.

${ }^{1}$ Hospital Universitário da Universidade Federal do Piauí (HU-UFPI), Teresina - PI.

*E-mail: pollyanaraujo@hotmail.com 


\section{RESUMEN}

Objetivo: Describir el uso de herramientas tecnológicas por parte de un Servicio de Vigilancia Epidemiológica Hospitalaria en el contexto de covid-19 en un Hospital Universitario del Nordeste de Brasil. Informe de experiencia: El Hospital Universitário do Piauí se ha convertido en un referente para covid-19 desde abril de 2020. Una vigilancia epidemiológica utiliza la recolección de datos a través de historias clínicas electrónicas y la comunicación con los pacientes y familiares mediante llamadas telefónicas para realizar la notificación. de los casos. Para la transferencia de información a organismos oficiales se utilizaron herramientas tecnológicas como E-SUS VE y Sivep Gripe. Con el fin de acceder a la información de que disponen los profesionales de la institución, se utiliza una intranet mediante Sisah, dispositivo mediante el cual se pudo difundir información relacionada con Notas Técnicas oficiales y actualizadas del Ministerio de Salud. Consideraciones finales: Los registros Las herramientas electrónicas y tecnológicas utilizadas en el cuidado de la salud tienen un gran potencial para mejorar la atención, facilitando la transmisión rápida y precisa de los datos de los pacientes, una razón extremadamente importante para las acciones de vigilancia epidemiológica de covid-19.

Palabras clave: Covid-19, Notificación, Innovación, Monitorización epidemiológica.

\section{INTRODUÇÃO}

Em dezembro de 2019 a Organização Mundial de Saúde (OMS) foi notificada pelas autoridades chinesas do surgimento de um no subtipo de coronavírus, o SARS-CoV-2. Em 30 de janeiro de 2020, a OMS declarou a epidemia do novo coronavírus uma emergência de saúde pública de importância internacional devido à velocidade com a qual o vírus se espalhava entre os continentes e, em 11 de março, a situação foi elevada pandemia (SOUZA DO, 2020).

O surgimento da covid-19 acarretou em mudanças drásticas na vida de toda população mundial. A doença destaca-se pela sua velocidade de disseminação e pelo seu alcance, os dados históricos recentes evidenciam essa dinâmica espaço-temporal (SOUSA DO, 2020). Diante desse cenário, diversas soluções têm sido propostas para o seu enfrentamento. Até o momento não existe vacina ou tratamento eficaz contra a covid19 (LIU J, et al., 2020).

Em todo o mundo, as medidas de isolamento social foram adotadas na tentativa de reduzir a propagação do SARS-COV-2. No entanto, é fundamental que os serviços de saúde possam antever os riscos para que assim, gerenciem ações preventivas e assistenciais nos casos da doença e dessa forma reduzam o número de óbitos (AYYOUBZADEH SM, et al., 2020).

Nesse contexto, a pandemia da covid-19 obrigou as instituições e os profissionais de saúde a inovarem os processos de trabalhos já anteriormente consolidados. Procedimentos cotidianos, como por exemplo, a paramentação dos profissionais, coleta de exames, procedimentos cirúrgicos, entre outros, tiveram que passar por reformulações diante da ameaça de disseminação do novo coronavírus.

A vigilância epidemiológica (VE), uma das mais antigas atividades de monitoramento em saúde, também passou por processos de inovações diante desse novo desafio da covid-19.Dentre as funções da VE pode se citar a orientação do Sistema Nacional de Vigilância em Saúde e a Rede de Serviços de Atenção à Saúde do SUS atuando na identificação e na notificação de casos suspeitos, confirmados e óbitos, na investigação laboratorial e no manejo adequado de casos por infecção humana pelo SARS-COV-2, de modo a reduzir os riscos de transmissão no território nacional (BRASIL, 2020a).

A inovação tecnológica em saúde pode ser concebida como a aplicação de novos conhecimentos, que aparecem na forma de artefato físico, ideias ou em novos procedimentos e práticas que trazem mudanças na assistência à saúde (BATISTA PCP, et al. 2011). Nesse sentido, o presente estudo tem por objetivo descrever a utilização de ferramentas tecnológicas por um Serviço de Vigilância Epidemiológica Hospitalar no contexto da covid-19 em um Hospital Universitário do Nordeste do Brasil. 


\section{RELATO DE EXPERIÊNCIA}

A experiência ocorreu em um hospital público de referência para covid-19 em Teresina, Piauí, de abril a setembro de 2020, período que este hospital se tornou referência no estado para a covid-19.

Em 19 de março de 2020 o Piauí confirmou seus primeiros casos e a partir de então, foram publicados decretos que determinavam medidas de isolamento social para o enfrentamento da crise provocada pela covid-19 no estado (SESAPI, 2020). A partir das pactuações e contratualizações, o Hospital Universitário da Universidade Federal do Piauí (HU-UFPI) configurou-se como um dos principais hospitais da rede de atenção à covid-19, chegando a ofertar 30 leitos de terapia intensiva e 38 de enfermarias clínica durante o pico de casos (MEC, 2020).

O HU-UFPI presta serviços de alta e média complexidade, com atendimento referenciado pela Central de Regulação de Leitos. Além disso, é um hospital escola, que recebe estudantes de diferentes cursos para as atividades de ensino, pesquisa e extensão (MEC, 2020).

Dentro do HU-UFPI a VE é o setor responsável por notificar os casos de covid-19, exercendo também a reponsabilidade de contribuir através da busca ativa na identificação de casos suspeitos, monitorar a realização de exames específicos, acompanhar os resultados, além de digitar as fichas de notificações em sistemas específicos.

Em consonância com a Portaria no 2.254/2010, a VE do HU-UFPI mantinha um sistema de busca ativa diária para os pacientes internados e atendidos na unidade hospitalar para a detecção das doenças e agravos de notificação, em especial, a covid-19, através do uso de sistemas internos da instituição, como o Sistema de Apoio a Administração Hospitalar (SISAH) e o Aplicativo de Gestão para Hospitais Universitários (AGHU), ferramentas tecnológicas que permitiram o alcance rápido e eficaz para visualização de exames solicitados e diagnósticos de pacientes atendidos em um determinado período de tempo.

Como mecanismo para notificações de casos suspeitos ou confirmados de covid-19, a VE utilizou a coleta de dados por meio do prontuário eletrônico e a comunicação com os pacientes e familiares por ligações telefônicas. O HU-UFPI conta com o AGHU que contém todas as informações registradas pelos profissionais da instituição sobre os pacientes atendidos. Trata-se de um prontuário eletrônico.

Após essa primeira coleta de dados por meio do AGHU, para complementação da ficha, era realizado o contato com familiares dos pacientes através de ligações telefônicas. A ligação tinha como objetivo a confirmação dos dados do prontuário eletrônico, a coleta de informações sobre os sinais e sintomas da covid19, os dados sobre vacinação, comorbidades e demais dados para o preenchimento da ficha de notificação. Essa ligação foi de suma importância para a completude dos campos essenciais das fichas notificadas e, dessa forma, para correta alimentação dos sistemas de monitoramentos da pandemia, como também, para a compreensão da situação epidemiológica, monitoramento dos casos, análises de riscos associado ao novo coronavírus.

Marques CA, et al. (2020) já citavam o quanto é imprescindível o estabelecimento de estratégias para a sensibilização e conscientização das equipes de saúde sobre importância da qualidade do registro das notificações compulsórias no Brasil, a partir do entendimento de que a falta informações contribui para o não conhecimento do real perfil epidemiológico e consequentemente, a dificuldade de avaliação da situação de agravos e das tomadas de decisões para controles das doenças notificadas.

Para esta ação de coletar as informações por ligações telefônicas também foi considerado os riscos de contaminação da equipe e a condições clínicas dos pacientes. Estes na sua maioria, encontravam-se em intubação orotraqueal ou em estado grave com a dependência da utilização de oxigênio e, assim, sem condições de comunicar-se efetivamente.

Essa estratégia foi primeiramente utilizada para pacientes mais graves. No entanto, com avanço no número dos casos e a sobrecarga da equipe de vigilância, a estratégia para de notificação de casos suspeitos e confirmados para Covid-19 por meios das tecnologias foi estendida também para pacientes de enfermaria que possuíam celulares e estavam em condições de comunicação. 
É importante destacar que nesse período, as recomendações dos órgãos reguladores de vigilância apontavam para a necessidade de restrições de profissionais assistindo pacientes suspeitos ou confirmados para a covid-19 (ANVISA, 2020). A falha na proteção aos profissionais de saúde é citada como uma forma de disseminação da doença. (SILVA AAM, 2020).

Nesse sentido as diversas ferramentas eletrônicas disponíveis contribuíram para facilitar o processo de notificação e acompanhamentos dos casos. Além do AGHU, outra ferramenta importante foi Gerenciador de Ambiente Hospitalar (GAL). O GAL é um sistema informatizado desenvolvido para os Laboratórios de Saúde Pública (LACEN) aplicado aos exames e ensaios com padrão nacional e desenvolvido de acordo com os protocolos do Ministério da Saúde (MS). A utilização desse sistema permite a VE gerenciar e acompanhar as realizações das análises laboratoriais, desde a sua solicitação até emissão do laudo final, de maneira ágil e fácil, e com isso subsidiar as tomadas de decisões por parte da VE de acordo com os resultados dos exames.

Diante do contexto mundial relativo à pandemia da covid-19, houve a necessidade de reorganização das rotinas dos cidadãos e de toda estruturação dos serviços de saúde do mundo inteiro. Tornou-se indispensável a elaboração de um plano emergencial de contingência, principalmente nos hospitais de referência da doença, para o manejo clínico dos pacientes e redução da transmissão intra-hospitalar. Ocorreu também a necessidade do surgimento de novos fluxos e processos assistenciais causando impacto nos colaboradores da saúde, pacientes e familiares.

Foi nesse sentido que a VE teve que se reinventar para ter condições de realizar todas as notificações de covid-19 dos pacientes atendidos com suspeita/confirmação da doença no HU-UFPI. Durante a pandemia, diminuir os riscos de contaminação dos profissionais nos hospitais foi um dos grandes desafios dos serviços de saúde. A equipe da VE desta instituição buscou novas formas de realizar as notificações dos casos.

A equipe da VE do HU-UFPI composta por médicos infectologistas, enfermeiros e técnicos em enfermagem direcionaram as condutas relacionadas à notificação e comunicação dos casos as autoridades competentes, monitoraram o seguimento das informações sobre a evolução dos casos acompanhados, incluindo a realização dos exames e resultados.

O serviço de VE do HU-UFPI conseguiu apropriar-se de sistemas do MS para a notificação de casos em tempo real. A utilização de sistemas como o E-SUS VE para notificações de síndrome gripal relacionada a covid-19 e o Sivep Gripe para notificações de casos de síndrome respiratória aguda grave proporcionou ao serviço agilidade e celeridade na comunicação da ocorrência de casos para as autoridades sanitárias. A covid19 é uma doença de notificação imediata com a comunicação da ocorrência de casos em até 24 horas (BRASIL, 2020b).

Os registros nos sistemas do MS auxiliam a gestão no monitoramento e na análise da situação epidemiológica da transmissão da covid-19, além de contribuir com a tomada de decisão acerca das medidas locais de isolamento social e nas ações de suporte da capacidade hospitalar instalada no município e nos serviços de referência da rede.

Outro grande desafio da VE foi a sobrecarga de trabalho da equipe. Muito se falou e mostrou nos meios de comunicação sobre a exaustão dos profissionais da linha de frente no combate ao Covid.19. No entanto, há de considerar também todos os demais profissionais envolvidos no trabalho em saúde que também tiveram que se adaptar a essa nova realidade.

Além do grande número de casos do novo coronavírus a serem notificados e monitorados, a VE também esteve envolvida em vários desafios relacionados a essa doença que são de sua responsabilidade, entre os quais, o controle dessa doença transmissível dentro do ambiente hospitalar. Ademais, as outras doenças de notificação seguem seu curso e precisam continuar sendo notificadas e monitoradas pela equipe da VE gerando assim a sobrecarga e exaustão também desses profissionais.

Para fins de acesso as informações disponíveis para os profissionais da instituição, utilizou-se a intranet através do uso do SISAH, dispositivo através do qual foi possível disseminar as informações relativas a Notas Técnicas oficiais e atualizadas do Ministério da Saúde; Orientações e normativas da Secretaria Estadual de 
Saúde do Piauí; Documentos normativos e com recomendações da Empresa Brasileira de Serviços Hospitalares (EBSERH), além de Protocolos Operacionais Padrão que direcionavam normas e condutas internas no âmbito do HU-UFPI. Segundo Xavier F, et al. (2020) a divulgação de informações atualizadas e confiáveis e de maneira rápida é dos grandes desafios da vigilância em saúde na pandemia com o advento das fakes news.

\section{DISCUSSÃO}

A estratégia de obtenção das informações por meio de ligações telefônicas mostrou-se bastante eficaz durante a pandemia da Covid-19. Um ponto a ser destacado foi como a maneira de comunicar-se com família, de forma empática e com linguagem simples, pode influenciar e facilitar a obtenção dos dados necessários. A realização de perguntas abertas como por exemplo, quais os sinais e sintomas que o paciente sentiu ou apresentou não se mostrou eficaz. Ao contrário, perguntas objetivas foram bem mais eficazes, como: $O$ paciente apresentou febre em algum momento? Apresentou diarreia? Queixou-se de dor de garganta? Com essas indagações simples e diretas pode se perceber que os familiares recordavam melhor sobre as informações requeridas.

O contato com familiares ainda que através de ligação de telefônica também aproximou o profissional da vigilância da realidade vivida pelas famílias durante a pandemia. Observou-se, por vezes, que os familiares tinham muitas dúvidas não somente sobre o estado de saúde do seu ente querido, mas também sobre a doença, sobre o que fazer com demais familiares que tiveram contato com paciente doente, onde procurar exames e tratamento. Realizar essas orientações foi mais uma função envolvida pela vigilância durante a pandemia.

Para Singhal T (2020) o indivíduo deve possuir um alto nível de participação no cuidado com a saúde, como ter conhecimento adequado da transmissão viral, engajar-se no autocuidado adequado, seguir diretrizes de auto-higiene e distanciamento social, fundamentais para evitar internações evitáveis. Entretanto, a pandemia da Covid-19 trouxe um incremento das vulnerabilidades e iniquidades sociais no ano de 2020 , com implicações em um acesso oportuno e na qualidade à saúde (PATEL JA, et al. 2020).

As ligações telefônicas para comunicação em saúde é uma estratégia que já vêm sendo utilizada no Brasil. O MS utilizou em iniciativas como o TeleSUS para realizar busca ativa de casos suspeitos de Covid-19, bem como para orientar as pessoas com sinais e sintomas de infecção por SARS-CoV-2 sem terem que sair de casa (CAETANO R, et al. 2020). A comunicação por telefone também foi utilizada pela PNAD covid-19, a Pesquisa Nacional por Amostra de Domicílios do IBGE (Instituto Brasileiro de Geografia e Estatística). A pesquisa objetivou estimar o número de pessoas com sintomas referidos associados à síndrome gripal e monitorar os impactos da pandemia no mercado de trabalho brasileiro (PENNA GO, et al.2020).

Ceccon RF e Schneider IJC (2020) consideram a utilização das tecnologias leves imprescindível para o enfrentamento dessa pandemia para o menor grau de adoecimento e morte.

A alta transmissibilidade da Covid-19 associada às várias formas de contágio até então descritas como contato direto e indireto, constituem alto risco de disseminação para a população. Devido a inexistência de medicamentos e vacina disponíveis, as estratégias de prevenção e controle da doença são essenciais neste momento da pandemia (WILDER-SMITH A e FREEDMAN DO, 2020). Evidenciou-se que a notificação de casos durante uma pandemia de uma doença com alto poder de disseminação através do prontuário eletrônico e por contato telefônico mostrou-se bastante satisfatória, demonstrando ainda como as tecnologias podem ser utilizadas para realização de processos tradicionais.

A experiência permitiu o desenvolvimento de habilidades de comunicação e cooperação pela equipe. A utilização de tecnologias e instrumentos facilitadores do manejo de informação entre os setores envolvidos permitiu a ampliação e o fortalecimento da vigilância epidemiológica no enfrentamento da pandemia. Deve incluir a interpretação dos autores sobre os resultados obtidos e sobre suas principais implicações, a comparação dos achados com a literatura, as limitações do estudo e eventuais indicações de caminhos para novas pesquisas. Cabe destacar que os processos e instrumentos utilizados permanecem em constante aprimoramento e adaptação por todos da equipe da epidemiologia hospitalar. 
Os registros eletrônicos e ferramentas tecnológicas utilizadas na saúde têm grande potencial para melhorar os cuidados, facilitando a transmissão rápida e precisa de dados do paciente, razão de extrema relevância para as ações de vigilância epidemiológica da covid-19, que, por vezes, necessita atuar de maneira segura, ágil e concisa em notificações imediatas e adoção de condutas fundamentais para o controle da doença. A aplicação da inovação de ações já realizadas cotidianamente acontece muitas vezes sem planejamento específico, mas que surpreende com os resultados alcançados. Cabe aos profissionais reconhecer e aprimorar essas ideias em benefício dos serviços.

\section{REFERÊNCIAS}

1. ANVISA. Agência Nacional de Vigilância Sanitária. Nota Técnica Anvisa 04/2020, atualizada em 27 de outubro de 2020. Orientações para Serviços de Saúde: Medidas de Prevenção e Controle que devem ser adotadas durante a assistência aos casos suspeitos ou confirmados de infecção pelo novo Coronavírus (SARS-CoV-2). Disponível em: file:///C:/Users/Pollyana/Downloads/NOTA_TECNICA_GVIMS_GGTES_ANVISA_04_2020_Reviso_27.10.2020.pdf. Acesso em: 29 nov. 2020.

2. AYYOUBZADEH SM, et al. Predicting COVID-19 Incidence Through Analysis of Google Trends Data in Iran: Data Mining and Deep Learning Pilot Study. JMIR Public Health Surveill, 2020; 6(2): e18828.

3. BATISTA PCP, et al. A inovação tecnológica como ferramenta para monitoramento da saúde dos trabalhadores de enfermagem. Revista da Escola de Enfermagem da USP, 2011; 45(Esp): 1621-1626.

4. BRASIL. Ministério da Saúde. Portaria No 2.254, de 05 de agosto de 2010 . Disponível em: http://bvsms.saude.gov.br/bvs/saudelegis/gm/2010/prt2254_05_08_2010.html. Acesso em: 20 out. 2020.

5. BRASIL. Ministério da Saúde. Secretaria de Vigilância em Saúde. Guia de Vigilância Epidemiológica - Emergências de Saúde Pública de Importância Nacional pela Doença pelo Novo Coronavírus 2019. v. 3. Brasília: Ministério da Saúde, 2020a.

6. BRASIL. Ministério da Saúde. Coronavírus (COVI-19). Governo Federal. 2020b. Disponível em: https://coronavirus.saude.gov.br/definicao-de-caso-e-notificacao. Acesso em: 19 out. 2020.

7. CAETANO R, et al. Desafios e oportunidades para telessaúde em tempos da pandemia pela COVID-19: uma reflexão sobre os espaços e iniciativas no contexto brasileiro. Cadernos de Saúde Pública, 2020; 36 (5): 1-16.

8. CECCON RF, SCHNEIDDER IJC. Tecnologias leves e educação em saúde no enfrentamento à pandemia da COVID19. Scielo Public Health; 2020; 1-19.

9. LIU J, et al. Community Transmission of Severe Acute Respiratory Syndrome Coronavirus 2, Shenzhen, China, 2020. Emerging Infectious Diseases. 2020; 26(6):1320-1323.

10. MARQUES CA, et al. Avaliação da não completude das notificações compulsórias de dengue registradas por município de pequeno porte no Brasil. Ciência \& Saúde Coletiva, 202025 (3): 891-900.

11. MINISTÉRIO DA EDUCAÇÃO (MEC). EBSERH. Hospitais Universitários Federais. Disponível em: http://www2.ebserh.gov.br/web/hu-ufpi/apresentacao. Acesso em: 5 set. 2020.

12. Organização Mundial da Saúde (OMS), Organização Pan-americana da saúde (OPAS). Folha informativa - COVID19 (doença causada pelo novo coronavírus). 2020.2 Disponível em: https://www.paho.org/bra/index.php?option=com_content\&view=article\&id=6101:covid 19\&ltemid=875. Acesso em: 05 out. 2020.

13. PATEL JA, et al. Poverty, inequality and COVID-19: the forgotten vulnerable. Public Health. 2020; 183:110-111.

14. PENNA GO, et al. PNAD COVID-19: um novo e poderoso instrumento para Vigilância em Saúde no Brasil. Ciências \& Saúde Coletiva, 2020; 25(9): 3567-3571.

15. Secretaria do Estado de Saúde do Piauí (SESAPI). Coronavírus Piauí. 2020. Governo do Estado do Piauí. Disponível em: http://coronavirus.pi.gov.br/. Acesso em: 20 out. 2020.

16. SILVA AAM. Sobre a possibilidade de interrupção da epidemia pelo coronavírus (COVID-19) com base nas melhores evidências científicas disponíveis. Revista Brasileira de Epidemiologia, 2020; 23: 1-3.

17. SOUZA DO. A pandemia de COVID-19 para além das Ciências da Saúde: reflexões sobre sua determinação social. Ciência \& saúde coletiva, 2020; 25 (supl.1): 2468-2477.

18. WILDER-SMITH A, FREEDMAN, DO. Isolation, quarantine, social distancing and community containment: pivotal role for old-style public health measures in the novel coronavirus (2019-nCoV) outbreak. Journal of Travel Medicine, 2020; 27(2): $1-4$.

19. XAVIER F, et al. Análise de redes sociais como estratégia de apoio à vigilância em saúde durante a Covid-19. Estudos avançados, 2020; 34 (99): 261-281.

20.SINGHAL T. Uma Revisão da Doença coronavírus-2019 (COVID-19). The Indian Journal of Pediatrics, 2020; 87(4): 281-286. 